\title{
REAL ZEROS OF A RANDOM SUM OF ORTHOGONAL POLYNOMIALS
}

\author{
MINAKETAN DAS
}

Abstract. Let $c_{0}, c_{1}, c_{2}, \cdots$ be a sequence of normally distributed independent random variables with mathematical expectation zero and variance unity. Let $P_{k}^{*}(x)(k=0,1,2, \cdots)$ be the normalised Legendre polynomials orthogonal with respect to the interval $(-1,1)$. It is proved that the average number of the zeros of $c_{0} P_{0}^{*}(x)+c_{1} P_{1}^{*}(x)+\cdots+c_{n} P_{n}^{*}(x)$ in the same interval is asymptotically equal to (3) $)^{-1 / 2} n$ when $n$ is large.

1. Let $\phi_{0}(x), \phi_{1}(x), \phi_{2}(x), \cdots$ be a sequence of polynomials orthogonal with respect to a given positive-valued weight function $\omega(x)$ over the interval $(a, b)$ where one or both of $a$ and $b$ may be infinite and let $\psi_{n}(x)=g_{n}^{-1 / 2} \phi_{n}(x)$ with

$$
g_{n}=\int_{a}^{b} \omega(x) \phi_{n}^{2}(x) d x .
$$

Let $f(x)$ be defined by

$$
f(x) \equiv f(\mathbf{c} ; x)=\sum_{k=0}^{N} c_{k} \psi_{k}(x),
$$

where the coefficients $c_{0}, c_{1}, c_{2}, \cdots$ form a sequence of mutually independent, normally distributed random variables with mathematical expectation zero and variance unity. We take the ordered set $c_{0}, \cdots, c_{n}$ as the point $\mathrm{c}$ in an $(n+1)$-dimensional real vector space $R_{n+1}$. The probability that the point $\mathrm{c}$ lies in an "infinitesimal rectangle" $\Pi(\mathbf{c})$ with sides of lengths $d c_{0}, d c_{1}, \cdots, d c_{n}$ is

$$
d P(\mathbf{c})=\prod_{k=0}^{n}\left\{(2 \pi)^{-1 / 2} \exp \left(-\frac{1}{2} c_{k}^{2}\right) d c_{k}\right\} .
$$

Let $N(c ; \alpha, \beta)$ denote the number of zeros of the polynomial (1.1) in the interval $\alpha \leqq x \leqq \beta$. We establish the formula

$$
\int_{R_{n+1}} N(\mathrm{c} ; \alpha, \beta) d P(\mathrm{c})=\frac{1}{\pi} \int_{\alpha}^{\beta}\left[\frac{S_{n}(x)+R_{n}(x)}{D_{n}(x)}-\frac{1}{4} \frac{Q_{n}^{2}(x)}{D_{n}^{2}(x)}\right]^{1 / 2} d x
$$

Received by the editors August 5, 1969.

AMS 1967 subject classifications. Primary 60XX, 6090.

Key words and phrases. Normally distributed random variables, mathematical expectation, variance, orthogonal polynomials.

Copyright (c) 1971, American Mathematical Society 
where

$$
\begin{aligned}
& D_{n}(x)=\phi_{n+1}^{\prime}(x) \phi_{n}(x)-\phi_{n+1}(x) \phi_{n}^{\prime}(x), \\
& Q_{n}(x)=\phi_{n+1}^{\prime \prime}(x) \phi_{n}(x)-\phi_{n+1}(x) \phi_{n}^{\prime \prime}(x), \\
& R_{n}(x)=\frac{1}{2}\left\{\phi_{n+1}^{\prime \prime}(x) \phi_{n}^{\prime}(x)-\phi_{n+1}^{\prime}(x) \phi_{n}^{\prime \prime}(x)\right\}
\end{aligned}
$$

and

$$
S_{n}(x)=\frac{1}{6}\left\{\phi_{n+1}^{\prime \prime \prime}(x) \phi_{n}(x)-\phi_{n+1}(x) \phi_{n}^{\prime \prime \prime}(x)\right\} .
$$

When $n$ is large, we can find an estimate of the integrand in the right-hand side of (1.2) in terms of $n$ and $x$ only in an easily integrable form, since only two functions $\phi_{n}(x)$ and $\phi_{n+1}(x)$ are now involved.

Let $P_{k}^{*}(x)$ be the normalized Legendre polynomial $\left(k+\frac{1}{2}\right)^{1 / 2} P_{k}(x)$, where

$$
P_{k}(x)=\frac{1}{2^{k}} \frac{1}{k !} \frac{d^{k}}{d x^{k}}\left(x^{2}-1\right)^{k}
$$

the famous Legendre polynomial. Here $a=-1$ and $b=1$ and $\omega(x) \equiv 1$. Further $\psi_{k}(x)=P_{k}^{*}(x)$ with $g_{n}=\left(n+\frac{1}{2}\right)^{1 / 2}$. We prove

THEOREM 1. The average number of zeros of

$$
c_{0} P_{0}^{*}(x)+c_{1} P_{1}^{*}(x)+\cdots+c_{k} P_{k}^{*}(x)+\cdots+c_{n} P_{n}^{*}(x)
$$

in $(-1,1)$ is asymptotically equal to $n / \sqrt{ } 3$ when $n$ is sufficiently large.

2. Let us put

$$
\begin{aligned}
& A \equiv A_{n}(x)=\psi_{0}^{2}(x)+\psi_{1}^{2}(x)+\cdots+\psi_{n}^{2}(x), \\
& B \equiv B_{n}(x)=\psi_{1}(x) \psi_{1}^{\prime}(x)+\cdots+\psi_{n}(x) \psi_{n}^{\prime}(x)
\end{aligned}
$$

and

$$
C \equiv C_{n}(x)=\left[\psi_{1}^{\prime}(x)\right]^{2}+\cdots+\left[\psi_{n}^{\prime}(x)\right]^{2} .
$$

Then, by Cauchy's inequality, $A C-B^{2} \geqq\left[\psi_{0} \psi_{1}^{\prime}\right]^{2}>0$. By proceeding as in $\$ 3$ of our earlier work (cf. [1]), we obtain

$$
\int_{R_{n+1}} N(c ; \alpha, \beta) d P(\mathrm{c})=\frac{1}{\pi} \int_{\alpha}^{\beta} \frac{\left(A C-B^{2}\right)^{1 / 2}}{A} d x .
$$

We put $\lambda_{n}=h_{n} g_{n}^{-1} h_{n+1}^{-1}$, where $h_{n}$ is the coefficient of $x^{n}$ in $\phi_{n}(x)$ and $g_{n}$ is defined as above. Then we have

$$
\sum_{k=0}^{n} g_{k}^{-1} \phi_{k}(x) \phi_{k}(y)=\lambda_{n} \frac{\phi_{n+1}(y) \phi_{n}(x)-\phi_{n+1}(x) \phi_{n}(y)}{y-x} .
$$


This is the famous Christofel-Darboux formula [3, p. 135] in the theory of orthogonal functions. We set $y=x+\delta$ in the formula (2.2) and equate the coefficients of like powers of $\delta$ on both sides to obtain

$$
\begin{aligned}
\sum_{\nu=0}^{n} g_{\nu}^{-1}\left[\phi_{\nu}(x)\right]^{2} & =\lambda_{n}\left[\phi_{n+1}^{\prime}(x) \phi_{n}(x)-\phi_{n+1}(x) \phi_{n}^{\prime}(x)\right], \\
\sum_{\nu=1}^{n} g_{\nu}^{-1}\left[\phi_{\nu}(x) \phi_{\nu}^{\prime}(x)\right] & =\frac{\lambda_{n}}{2}\left[\phi_{n+1}^{\prime \prime}(x) \phi_{n}(x)-\phi_{n+1}(x) \phi_{n}^{\prime \prime}(x)\right]
\end{aligned}
$$

and

$$
\sum_{\nu=1}^{n} g_{\nu}^{-1}\left[\phi_{\nu}(x) \phi_{\nu}^{\prime \prime}(x)\right]=\frac{\lambda^{n}}{3}\left[\phi_{n+1}^{\prime \prime \prime}(x) \phi_{n}(x)-\phi_{n+1}(x) \phi_{n}^{\prime \prime \prime}(x)\right]
$$

Differentiating (2.4) and making use of (2.5), we get

$$
\begin{aligned}
\sum_{\nu=1}^{n} g_{\nu}^{-1}\left[\phi_{\nu}^{\prime}(x)\right]^{2}= & \frac{\lambda_{n}}{6}\left[\phi_{n+1}^{\prime \prime \prime}(x) \phi_{n}(x)-\phi_{n+1}(x) \phi_{n}^{\prime \prime \prime}(x)\right] \\
& +\frac{\lambda_{n}}{2}\left[\phi_{n+1}^{\prime \prime}(x) \phi_{n}^{\prime}(x)-\phi_{n+1}^{\prime}(x) \phi_{n}^{\prime \prime}(x)\right] .
\end{aligned}
$$

Making use of (2.3), (2.4) and (2.6), and the fact that $\lambda_{n} \neq 0$, we obtain the formula (1.2).

3. For Legendre polynomials $P_{n}(x)$, we have the relations

$$
\left(1-x^{2}\right) P_{n+1}^{\prime \prime}(x)=2 x P_{n+1}^{\prime}(x)-(n+1)(n+2) P_{n+1}(x)
$$

and

$$
\left(1-x^{2}\right) P_{n}^{\prime \prime}(x)=2 x P_{n}^{\prime}(x)-n(n+1) P_{n}(x) .
$$

From (3.1) and (3.2), we obtain

$$
\begin{aligned}
& \left(1-x^{2}\right)\left[P_{n+1}^{\prime \prime}(x) P_{n}^{\prime}(x)-P_{n}^{\prime \prime}(x) P_{n+1}^{\prime}(x)\right] \\
& =-(n+1)\left[n\left\{P_{n+1}(x) P_{n}^{\prime}(x)-P_{n}(x) P_{n+1}^{\prime}(x)\right\}+2 P_{n+1}(x) P_{n}^{\prime}(x) \mid\right.
\end{aligned}
$$

and

$$
\begin{aligned}
& \left(1-x^{2}\right)\left[P_{n+1}^{\prime \prime}(x) P_{n}(x)-P_{n+1}(x) P_{n}^{\prime \prime}(x)\right] \\
& \quad=2 x\left[P_{n+1}^{\prime}(x) P_{n}(x)-P_{n+1}(x) P_{n}^{\prime}(x)\right]-2(n+1) P_{n}(x) P_{n+1}(x) .
\end{aligned}
$$

Differentiating (3.4) and using (3.3), we get 


$$
\begin{aligned}
& \left(1-x^{2}\right)\left[P_{n+1}^{\prime \prime \prime}(x) P_{n}(x)-P_{n}^{\prime \prime \prime}(x) P_{n+1}(x)\right] \\
& =(n+1)\left[n\left\{P_{n+1}(x) P_{n}^{\prime}(x)-P_{n}(x) P_{n+1}^{\prime}(x)\right\}+2 P_{n+1}(x) P_{n}^{\prime}(x)\right] \\
& \quad+\frac{16}{1-x^{2}}\left[x\left\{P_{n+1}^{\prime}(x) P_{n}(x)-P_{n}^{\prime}(x) P_{n+1}(x)\right\}\right. \\
& \left.-(n+1) P_{n}(x) P_{n+1}(x)\right] \\
& \quad+2(n+1)\left[P_{n}^{\prime}(x) P_{n+1}(x)-P_{n}(x) P_{n+1}^{\prime}(x)\right] .
\end{aligned}
$$

We recall another formula for the derivative of a Legendre function $[2$, p. 179, (17)], viz.:

$$
\left(x^{2}-1\right) P_{n}^{\prime}(x)=n x P_{n}(x)-n P_{n-1}(x)
$$

and

$$
\left(x^{2}-1\right) P_{n+1}^{\prime}(x)=(n+1) x P_{n+1}(x)-(n+1) P_{n}(x)
$$

The application of (3.6) and (3.7) yields

$$
\begin{aligned}
& \left(x^{2}-1\right)\left[P_{n+1}^{\prime}(x) P_{n}(x)-P_{n+1}(x) P_{n}^{\prime}(x)\right] \\
& =(n+1)\left[2 x P_{n}(x) P_{n+1}(x)-P_{n}^{2}(x)-P_{n+1}^{2}(x)\right] \\
& \left(x^{2}-1\right)\left[P_{n+1}^{\prime}(x) P_{n}(x)+P_{n+1}(x) P_{n}^{\prime}(x)\right] \\
& =(n+1)\left[P_{n+1}^{2}(x)-P_{n}^{2}(x)\right]
\end{aligned}
$$

and

$$
\left(x^{2}-1\right) P_{n+1}(x) P_{n}^{\prime}(x)=(n+1) P_{n+1}(x)\left[P_{n+1}(x)-x P_{n}(x)\right]
$$

To evaluate

$$
P_{n}^{2}(x)+P_{n+1}^{2}(x)-2 x P_{n}(x) P_{n+1}(x),
$$

we set $x=\cos \gamma$ and make use of the celebrated Laplace's formula (cf. $\left[2\right.$, p. 208]) giving the asymptotic value of $P_{n}(\cos \gamma)$ as

$$
\left(\frac{2}{\pi n \sin \gamma}\right)^{1 / 2} \cos \left[\left(n+\frac{1}{2}\right) \gamma-\frac{\pi}{4}\right]+O\left((n \sin \gamma)^{-3 / 2}\right)
$$

in the range $\epsilon<\gamma<\pi-\epsilon$, where $0<\epsilon<\pi / 2$. After some simplifications, we find 


$$
\begin{aligned}
P_{n}^{2}(x)+ & P_{n+1}^{2}(x)-2 x P_{n}(x) P_{n+1}(x) \\
= & \frac{2}{\pi n \sin \gamma}\left\{\cos ^{2}\left[\left(n+\frac{1}{2}\right) \gamma-\frac{\pi}{4}\right]+\cos ^{2}\left[\left(n+\frac{3}{2}\right) \gamma-\frac{\pi}{4}\right]\right. \\
& \left.\quad-2 \cos \gamma \cos \left[\left(n+\frac{1}{2}\right) \gamma-\frac{\pi}{4}\right] \cos \left[\left(n+\frac{3}{2}\right) \gamma-\frac{\pi}{4}\right]\right\} \\
& +O\left(n^{-2} \operatorname{cosec}^{2} \gamma\right) \\
= & \frac{2}{\pi n}\left(1-x^{2}\right)^{1 / 2}+O\left(n^{-2}\left(1-x^{2}\right)^{-1}\right) .
\end{aligned}
$$

Making use of (3.8), we obtain

$$
\left\{P_{n+1}^{\prime}(x) P_{n}(x)-P_{n+1}(x) P_{n}^{\prime}(x)\right\}>\frac{2}{\pi}\left(1-x^{2}\right)^{-1 / 2}
$$

for sufficiently large $n$ and $|x|<1-n^{-2 / 3} \log n$. By the first theorem of Stieltjes, $[2$, p. $197,(8)]\left|P_{n}(x)\right| \leqq 4 n^{-1 / 2}\left(1-x^{2}\right)^{-1 / 4}$ and by (3.6), $\left|P_{n}^{\prime}(x)\right| \leqq 8 n^{1 / 2}\left(1-x^{2}\right)^{-5 / 4}$. Thus

$$
\begin{aligned}
n P_{n}(x) P_{n+1}(x) & =O\left(\left(1-x^{2}\right)^{-1 / 2}\right) \\
P_{n}(x) P_{n}^{\prime}(x) & =O\left(\left(1-x^{2}\right)^{-3 / 2}\right)
\end{aligned}
$$

and

$$
P_{n+1}^{\prime}(x) P_{n}(x)+P_{n}^{\prime}(x) P_{n+1}(x)=O\left(\left(1-x^{2}\right)^{-3 / 2}\right) .
$$

By putting these estimates in (3.3), (3.4) and (3.5), we get

$$
\begin{aligned}
& \left(1-x^{2}\right)\left(P_{n+1}^{\prime \prime} P_{n}^{\prime}-P_{n}^{\prime \prime} P_{n+1}^{\prime}\right) \\
& =n(n+1)\left(P_{n+1}^{\prime} P_{n}-P_{n}^{\prime} P_{n+1}\right)+O\left(n\left(1-x^{2}\right)^{-3 / 2}\right) \\
& \left(1-x^{2}\right)\left(P_{n+1}^{\prime \prime} P_{n}-P_{n}^{\prime \prime} P_{n+1}\right) \\
& =2 x\left(P_{n+1}^{\prime} P_{n}-P_{n}^{\prime} P_{n+1}\right)+O\left(\left(1-x^{2}\right)^{-3 / 2}\right)
\end{aligned}
$$

and

$$
\begin{aligned}
\left(1-x^{2}\right)\left(P_{n+1}^{\prime \prime \prime} P_{n}-P_{n}^{\prime \prime \prime} P_{n+1}\right)= & \left\{\frac{16 x}{1-x^{2}}-n-n^{2}\right\}\left(P_{n+1}^{\prime} P_{n}-P_{n}^{\prime} P_{n+1}\right) \\
& +O\left(n\left(1-x^{2}\right)^{-3 / 2}\right),
\end{aligned}
$$


where we have written $P_{k}, P_{k}^{\prime}, P_{k}^{\prime \prime}$ and $P_{k}^{\prime \prime \prime}$ for $P_{k}(x), P_{k}^{\prime}(x), P_{k}^{\prime \prime}(x)$ and $P_{k}^{\prime \prime \prime}(x)$, respectively. This abbreviation is also employed below.

By using (3.11), we finally obtain, for $|x|<1-n^{-2 / 3} \log n$, the estimate

$$
\begin{aligned}
& \left(P_{n+1}^{\prime \prime \prime} P_{n}-P_{n+1} P_{n}^{\prime \prime \prime}\right) /\left(P_{n+1}^{\prime} P_{n}-P_{n+1} P_{n}^{\prime}\right)=-n^{2}\left(1-x^{2}\right)^{-1}(1+O(1 / n)), \\
& \left(P_{n+1}^{\prime \prime} P_{n}^{\prime}-P_{n+1}^{\prime} P_{n}^{\prime \prime}\right) /\left(P_{n+1}^{\prime} P_{n}-P_{n+1} P_{n}^{\prime}\right)=n^{2}\left(1-x^{2}\right)^{-1}(1+O(1 / n))
\end{aligned}
$$

and

$$
\left(P_{n+1}^{\prime \prime} P_{n}-P_{n+1} P_{n}^{\prime \prime}\right) /\left(P_{n+1}^{\prime} P_{n}-P_{n+1} P_{n}^{\prime}\right)=O\left(n^{2 / 3}\left(1-x^{2}\right)^{-1}\right) .
$$

Putting these values for $Q_{n}(x) / D_{n}(x), R_{n}(x) / D_{n}(x)$ and $S_{n}(x) / D_{n}(x)$ in (1.2), the expression enclosed by brackets is estimated by

$$
\frac{n^{2}}{3} \frac{1}{\left(1-x^{2}\right)}\left[1+O\left(\frac{1}{(\log n)^{3}}\right)\right] \text {. }
$$

Let $\epsilon=n^{-2 / 3} \log n$ and $N(c ; \epsilon)$ denote the number of zeros of

$$
f(c ; x)=c_{0} P_{0}^{*}(x)+c_{1} P_{1}^{*}(x)+\cdots+c_{n} P_{n}^{*}(x)
$$

in $-1+\epsilon \leqq x \leqq 1-\epsilon$. By (1.2), we have

$$
\begin{aligned}
\int_{R_{n+1}} N(\mathrm{c} ; \epsilon) d P(\mathrm{c}) & =\frac{1}{\pi} \int_{-1+\epsilon}^{1-\epsilon} \frac{n}{\sqrt{ } 3}\left[1+O\left((\log n)^{-3}\right)\right]\left(1-x^{2}\right)^{-1 / 2} d x \\
& =\frac{n}{\sqrt{ } 3}\left\{1+O\left((\log n)^{-3}\right)\right\} .
\end{aligned}
$$

To complete the proof of Theorem 1, we observe (cf. $[2$, p. 250]) that

$$
\left|P_{n}(z)\right|=\frac{1}{\pi}\left|\int_{0}^{\pi}\left\{z+i\left(1-z^{2}\right)^{1 / 2} \cos \tau\right\}^{n} d \tau\right|
$$

and thus for $z=1+\epsilon e^{i \theta},\left|P_{n}(z)\right|<(1+3 \epsilon)^{n}<2 n^{3} \exp \left(n^{1 / 3}\right)$. Further $P_{n}(1)=1$. We can prove, as in our work $[1$, p. 722$]$, that

$$
\operatorname{Pr}\left(\max _{0 \leqq k \leqq n}\left|c_{k}\right| \leqq n\right)>1-e^{-n / 3},
$$

so that for $z=1+\epsilon e^{i \theta}$, we have

$$
\left(\operatorname{Pr}\left(|f(c ; z)| \geqq 4 n^{4} \exp \left(n^{1 / 3}\right)\right)\right)<\exp \left(-n^{2} / 3\right)
$$

and 


$$
(\operatorname{Pr}(|f(c ; z)|<1))<1 / n .
$$

Let $\nu(c ; \epsilon)$ denote the number of zeros of $|f(c ; z)|$ in $|z-1| \leqq \epsilon$. By making use of Jensen's theorem, we find

$$
\nu(\mathrm{c} ; \epsilon) \log 2 \leqq \frac{1}{2 \pi} \int_{0}^{2 \pi} \log \left|\frac{f\left(\mathrm{c} ; 1+\epsilon e^{i \theta}\right)}{f(\mathrm{c} ; 1)}\right| d \theta=O\left(n^{1 / 3}\right)
$$

with probability at least equal with $1-2 / n$. This shows that the average number of zeros of $f(\cdot ; x)$ in $1-\epsilon \leqq x \leqq 1$ and similarly in $-1 \leqq x \leqq-1+\epsilon$ is $O\left(n^{1 / 3}\right)$. Therefore, on using (3.16), we finally obtain the proof of Theorem 1 .

\section{REFERENCES}

1. M. Das, The average number of real zeros of a random trigonometric polynomial, Proc. Cambridge Philos. Soc. 64 (1968), 721-729. MR 38 \#1720.

2. G. Sansone, Orthogonal functions, Zanichelli, Bologna, 1952; English transl., Pure and Appl. Math., vol. 9, Interscience, New York, 1959. MR 13, 741; MR 21 \#2140.

3. E. D. Rainville, Special functions, Macmillan, New York, 1960. MR 21 \#6447.

Fakir Mohan College, Balasore, Orissa, India 